\title{
Factors Influencing the Happiness of Late School-aged Children: A Focus on Family Strength and Self-control
}

\author{
Bo Kyoung Jin ${ }^{1}$, Hye Young $\mathrm{Ahn}^{2}$ \\ ${ }^{1}$ Graduate Student, Graduate School, Department of Nursing, Eulji University, Daejeon; ${ }^{2}$ Professor, College of Nursing, Eulji University, Daejeon, Korea
}

\begin{abstract}
Purpose: The purpose of this study was to obtain research-based evidence on the relationships among general characteristics, family strength, self-control, and happiness among late school-aged children using a correlational research design. Methods: The participants were 172 fifth- and sixth-grade students from two public elementary schools. Data were collected by employing structured questionnaires, including the Korean Family Strengths Scale for Strengthening Family II, a self-control scale, and a happiness scale. Data analysis was conducted using SPSS version 23.0. Results: The level of happiness of late school-aged students showed significant correlations with family strength $(\mathrm{r}=.78, p<.001)$, and self-control $(\mathrm{r}=.59, p<.001)$. Family strength had a significant positive correlation with self-control $(\mathrm{r}=.55, p<.001)$. The factors with a significant impact on participants' happiness were family strength $(\beta=.63, p<.001)$, self-control $(\beta=.21, p<.001)$, exercise frequency, and self-perceived health. The total explanatory power of the model was $69 \%$, and the explanatory power of family strength for the level of happiness was $61 \%$, showing that the family strength was the most important factor that promoted happiness in late school-aged students. Conclusion: These findings imply that improving family strength is an important aspect of promoting happiness among late school-aged children. Interventions to strengthen late school-aged children's self-control are also necessary.
\end{abstract}

Key words: Happiness; Children; Family; Self-control

\section{Corresponding author Hye Young Ahn}

https://orcid.org/0000-0002-5353-671X

College of Nursing, Eulji University,

771-77 Beon-gil, Gyeryong-ro, Joong-gu, Daejeon 34824, Korea

TEL +82-42-259-1715 FAX +82-42-259-1709

E-MAIL ahanaya@eulji.ac.kr

*This article was adapted from a thesis by Bo Kyoung Jin in partial fulfillment of the requirements for the master's degree at Eulji University.

Received Feb 4, 2019 Revised Apr 22, 2019 Accepted Jun 4, 2019 (a) This is an Open Access article distributed under the terms of the Creative Commons Attribution NonCommercial License (http://creativecommons.org/licenses/by-nc/4.0/) which permits unrestricted noncommercial use, distribution, and reproduction in any medium, provided the original work is properly cited.

\section{INTRODUCTION}

\section{Need for Study}

The transition to modern society has caused numerous major problems relating to children's health, including some emerging problems with a negative effect on children's development such as poverty, dropping out of school, violence, and family breakdown [1]. As a reflection of common mental health problems in adolescence, suicide remains the top cause of deathamong adolescents (aged 9 24) in South Korea (hereafter, Korea), although the number of adolescent suicides has slightly decreased [2]. Even if serious psychological problems develop during childhood, the pursuit of a happy life is a common goal of human existence [3]. Happiness affects both lifespan and the quality of life. People who reported feeling very happy lived longer and had a lower risk of death than those who were less likely or unlikely to feel happy [4].

Comparative international research found that children and adolescents in Korea scored 90.4 (out of an average score of 100 for Organization for Economic Co-operation and Development countries) for subjective happiness; this score has increased in recent years, but remains lower than that of other countries [5]. Happiness is defined as a state in which people experience a lower of negative sentiment, a high level of positive emotion, 
and a high level of satisfaction with valuable work or activities that contribute to the development of society and individuals [6]. A 70-year longitudinal study on happiness, conducted by Harvard University, suggested that happy and healthy senescence depended on social human relations, not on high intellectual levels or social class [3]. The need for human relation forms a fundamental and crucial motivation for human beings [7]. Experiencing happiness and satisfaction by meeting one's needs for relationships is an important aspect of life [7]. In other words, good relationships may improve one's awareness of social support, reduce depression and anxiety, and increase happiness [6]. Therefore, the emotional support received from other people in human relationships is a stronger determinant of happiness than household socioeconomic status, academic performance, or self-efficiency [8].

Kang suggested that four components of happiness-self-esteem, optimism, peer relations, and the home environmentwere relevant for elementary school children in Korea [6]. Of these four components, strong human relations (encompassing both peer relations and parent-child relations) and emotional support (e.g., a sense of stability) had a particularly strong impact on childrens' perceptions of happiness [8]. School-aged children can perceive support and satisfaction as members of society when they experience better relationships through a healthy family [1].

In the same context, human relations that lead to happiness may involve family strength as a factor that provides a sense of stability and emotional support. Although research on the family, which is the most universal and important element of human relations that contributes to happiness among schoolaged children, has generally focused on problems and pathology in family life, discussions since the 1960s have also explored how to reinforce the positive aspects and strengths of families and investigated way of improving family relations [9]. Family strength can be defined as a framework that promotes the healthy development of individual members of the family, facilitates the ability of family members to share a value system as a group due to harmonious familiar interaction, and contributes to smooth interactions with kinship and social systems $[10,11]$.

Since the 1990s, research into healthy families has been conducted in Korea [12,13]. Children and adolescents spend a large amount of time with their family, and have many experiences at home. Family-centered care is also valued for pediatrics inpatients, in terms of both daily life and diseases management [14]. Human beings, who are independent, are affected by their family, which forms their primary environment, as they establish their personal identity. Therefore, the more important human beings' internal needs or establishment of a personal identity becomes, the more necessary and important it becomes to have a healthy family [12].

In addition, self-control is the ability to refrain from impulsive desires and behaviors and to delay pleasure and satisfaction [15]. This refers to the ability to behave in a way that is suitable for given situation and the self-regulation to control and delay the pursuit of immediate and impulsive satisfaction in pursuit of better outcomes and satisfaction [16]. Self-control starts with interactive control between the mother and the newborn infant, and it is important to emphasize the role of nature and nurture in children's development of self-control [17]. When parents treat their child's impulsive reactions carefully and consistently, the child develops better self-control [18]. It is therefore necessary to regard self-control, which facilitates impulse control and leads to long-term satisfaction, as a major variable when analyzing happiness among schoolaged children.

Philosophical thinking about happiness started in ancient times, and the psychological aspect of the role of nurses involved in children's physical and mental health has become more important in our rapidly-changing society [1]. From the perspective of personal health and healing, explorations of happiness in the practical and theoretical development of nursing should reflect subjects' values regarding the concept of health. It is therefore necessary to consider and encourage personal values, including human happiness, throughout nursing as a holistic discipline [19].

While the theme of happiness has long been investigated from various points of view, it is necessary to conduct basic nursing research on this topic, since little nursing research has investigated family strength and self-control. Preadolescence is the period that begins toward the end of childhood and ends at the 13th birthday. Children between 11 and 12 years old are generally considered to be preadolescents. This is a time when the developmental features of childhood and early adolescence overlap considerably [20]. This study aimed to investigate the factors influencing happiness among fifth- and sixth-graders, an age of transition into adolescence.

\section{Objectives}

This study aimed to investigate family strength, self-control, and happiness, as perceived by elementary school children, with the goal of identifying the factors affecting their happiness.

\section{METHODS}

\section{Study Design}

This study involved descriptive correlational research to in- 
vestigate family strength, self-control, and happiness among late school-aged children and to provide basic data that can be used to help develop nursing interventions regarding their psychological and emotional well-being.

\section{Subjects and Research Duration}

The data were collected from June to July 2016. Fifth-and sixth-graders who understood the purpose of this study participated, at two elementary schools in D Metropolitan City. G*Power version 3.1.9.2 was used to estimate the sample size for linear multiple regression. A total minimum sample size of 171 persons was found, with an effect size $\left(\mathrm{f}^{2}\right)$ of .10 , a significance level $(\alpha)$ of .05 , power $(1-\beta)$ of .90 , and five predictors [21]. We distributed 320 copies and collected 244 returned surveys, of which 72 were excluded as containing insincere answers and 172 were analyzed. Therefore, the response rate was $76 \%$.

\section{Instruments}

\section{1) Family strength}

To measure children's perceptions of family strength, the Korean Family Strength Scale for Strengthening Family II developed by Yoo et al. [13] was used. This tool contains a total of 22 items to measure such sub-concepts of family resilience (7 questions), mutual respect and acceptance (5 questions), qualitative bonding (4 questions), economic stability and cooperation (3 questions), and family cultural and social participation (3 questions). Each item is measured on a 5-point Likert scale with scores ranging from 1 ("totally disagree") to 5 ("totally agree"). Higher scores indicate greater family strength. For internal consistency reliability, Cronbach's $\alpha$ was .94 at the time of its development [13] and .95 in this study.

\section{2) Self-control}

We obtained permission to use the tools developed by Nam and Ok [18], and Koo [22]. We confirmed the items and confirmed their face validity through a pilot study. An adapted scale with a total of 20 items as used to measure children's self-control. The items in this tool are related to the pursuit of long-term (10 questions) and immediate (10 questions) satisfaction. Each item is measured on a 5-point Likert scale with scores ranging from 1 ("totally disagree") to 5 ("totally agree”). Higher scores indicate greater self-control. For internal consistency reliability, Cronbach's $\alpha$ was .78 in previous research [18] and .87 in this study.

\section{3) Happiness}

To measure children's perceptions of happiness, the scale developed by Kang [6] with a total of 36 items was used. This tool contains the sub-factors of self-esteem (12 questions), optimism (10 questions), peer relations (6 questions), and home environment (8 questions). Each item is measured on a 5-point Likert scale with scores ranging from 1 ("totally disagree") to 5 ("totally agree"). Higher scores indicate more happiness. For internal consistency reliability, Cronbach's $\alpha$ was .95 at the time of its development [6] and .97 in this study.

\section{4) General characteristics}

The questionnaire included items on gender and grade [23], parents' education, religion [18], economic level [18,23], selfperceived health [12], being alone [20], time spent conversing with parents, time spent using the internet [24], and exercise frequency [25].

General characteristics were indicated by selecting a given category or by writing a response directly. Gender was indicated as male or female. Respondents indicated their own grade. Parents' educational background was classified as middle school graduation or lower, high school graduation, college graduation, graduate school graduation. Perceived economic status was categorized as high, middle or low. Selfperceived health was classified as healthy, moderate or poor. For time spent conversing with parents, the categories were $<30 \mathrm{~min} /$ day, 30 59 $\mathrm{min} /$ day, and $\geq 60 \mathrm{~min} /$ day. For time spent on the internet, the categories were $<60 \mathrm{~min} /$ day, 60 $119 \mathrm{~min} /$ day, and $\geq 120 \mathrm{~min} /$ day. Exercise frequency was categorized as $<1,1 \sim 2,3 \sim 4$, and $\geq 5$ times per week.

\section{Data Collection}

The data were collected after obtaining approval from the Institutional Review Board (**16-25). Information about the researcher was given to the vice-principals and head teachers in charge of the fifth and sixth grades at two elementary schools in D Metropolitan City. The provided information included the purpose of the research and the data collection methods, confidentiality, possible advantages and disadvantages of participation, and a promise not to use the responses for any other purpose than that of the research. Participants were also informed that they could discontinue participation at any time by refusing to complete the questionnaire during the data collection process. Parents and students were asked to provide written consent before the study was conducted. At the request of the school, the questionnaire was distributed to all students in the fifth and sixth grades at the elementary schools.

\section{Data Analysis}

The collected data were analyzed using SPSS version 23.0 
(IBM Corp., Armonk, NY, USA). Participants' general characteristics, family strength, happiness, and self-control were analyzed using descriptive statistics. The differences in family strength, happiness, and self-control according to the general characteristics of the late school-aged children were analyzed using the $\mathrm{t}$-test and analysis of variance. Post hoc testing was done using the Scheffé test. The correlations among family strength, happiness, and self-control, as perceived by the late school-aged children, were analyzed using Pearson' correlation coefficients. The effects of these factors on their happiness were analyzed using stepwise multiple regression.

\section{RESULTS}

\section{Family Strength, Self-control, and Happiness by General Characteristics}

The mean scores for family strength, self-control, and hap- piness by general characteristics are presented in Table 1 . The following significant differences in mean family strength scores according to the general characteristics were found. Sixth-graders scored significantly higher than fifth-graders $(\mathrm{t}=2.15, p=.033)$. Children whose mothers were college graduates or had a higher education level had significantly higher family strength scores than those whose mothers were high school graduates or lower $(t=3.22, p=.002)$. Children with a high economic status scored highest for family strength, followed by those at the middle and low levels $(\mathrm{F}=6.99, p=.001)$. Family strength differed significantly by self-perceived health $(\mathrm{F}=3.81, p=.024)$. The Scheffé test found that children who perceived themselves to be very healthy had higher family strength scores than those with moderate self-perceived health. Family strength differed significantly according to the amount of time spent conversing with parents ( $\mathrm{F}=28.04, p<$ .001 ), with the highest scores found for those who reported $\geq 60 \mathrm{~min} /$ day of conversation, followed by those who re-

Table 1. Family Strength, Self-control, and Happiness according to General Characteristics

$(N=172)$

\begin{tabular}{|c|c|c|c|c|c|c|c|c|}
\hline \multirow[b]{2}{*}{ Variable } & \multirow[b]{2}{*}{ Categories } & \multirow[b]{2}{*}{$\mathrm{n}$} & \multicolumn{2}{|c|}{ Family strength } & \multicolumn{2}{|c|}{ Self-control } & \multicolumn{2}{|c|}{ Happiness } \\
\hline & & & $\mathrm{M} \pm \mathrm{SD}$ & $\begin{array}{l}\text { t or F }(p) \\
\text { Scheffé* }\end{array}$ & $\mathrm{M} \pm \mathrm{SD}$ & $\begin{array}{l}\text { t or F }(p) \\
\text { Scheffé* }^{*}\end{array}$ & $\mathrm{M} \pm \mathrm{SD}$ & $\begin{array}{l}\text { t or F }(p) \\
\text { Scheffé* }\end{array}$ \\
\hline Gender & $\begin{array}{l}\text { Male } \\
\text { Female }\end{array}$ & $\begin{array}{r}66 \\
106\end{array}$ & $\begin{array}{l}82.09 \pm 15.14 \\
82.77 \pm 15.64\end{array}$ & $\begin{array}{l}0.28 \\
(.778)\end{array}$ & $\begin{array}{l}70.30 \pm 11.05 \\
72.57 \pm 11.04\end{array}$ & $\begin{array}{l}1.31 \\
(.193)\end{array}$ & $\begin{array}{l}139.98 \pm 24.69 \\
138.09 \pm 24.14\end{array}$ & $\begin{array}{l}0.50 \\
(.621)\end{array}$ \\
\hline Grade & $\begin{array}{l}\text { Fifth } \\
\text { Sixth }\end{array}$ & $\begin{array}{r}101 \\
71\end{array}$ & $\begin{array}{l}80.42 \pm 15.72 \\
85.49 \pm 14.55\end{array}$ & $\begin{array}{l}2.15 \\
(.033)\end{array}$ & $\begin{array}{l}68.92 \pm 11.24 \\
75.65 \pm 9.59\end{array}$ & $\begin{array}{c}4.10 \\
(<.001)\end{array}$ & $\begin{array}{l}134.73 \pm 25.22 \\
144.63 \pm 21.81\end{array}$ & $\begin{array}{l}2.68 \\
(.008)\end{array}$ \\
\hline Father's education $^{\dagger}$ & $\begin{array}{l}\leq \text { High school } \\
\geq \text { College }\end{array}$ & $\begin{array}{l}52 \\
89\end{array}$ & $\begin{array}{l}82.98 \pm 16.78 \\
85.02 \pm 14.95\end{array}$ & $\begin{array}{l}0.75 \\
(.456)\end{array}$ & $\begin{array}{l}70.65 \pm 11.09 \\
74.91 \pm 10.08\end{array}$ & $\begin{array}{l}2.33 \\
(.021)\end{array}$ & $\begin{array}{l}140.42 \pm 24.72 \\
142.82 \pm 23.39\end{array}$ & $\begin{array}{l}0.58 \\
(.566)\end{array}$ \\
\hline $\begin{array}{l}\text { Mother's } \\
\text { education }^{\dagger}\end{array}$ & $\begin{array}{l}\leq \text { High school } \\
\geq \text { College }\end{array}$ & $\begin{array}{l}58 \\
82\end{array}$ & $\begin{array}{l}79.21 \pm 15.96 \\
87.56 \pm 14.47\end{array}$ & $\begin{array}{l}3.22 \\
(.002)\end{array}$ & $\begin{array}{l}72.34 \pm 11.32 \\
73.79 \pm 10.15\end{array}$ & $\begin{array}{l}0.79 \\
(.429)\end{array}$ & $\begin{array}{l}139.02 \pm 23.17 \\
143.72 \pm 24.45\end{array}$ & $\begin{array}{l}1.15 \\
(.254)\end{array}$ \\
\hline $\begin{array}{l}\text { Economic } \\
\text { status }\end{array}$ & $\begin{array}{l}\text { High }^{\mathrm{a}} \\
\text { Middle }^{\mathrm{b}} \\
\text { Low }^{\mathrm{c}}\end{array}$ & $\begin{array}{r}44 \\
114 \\
14\end{array}$ & $\begin{array}{l}88.30 \pm 11.82 \\
81.56 \pm 15.89 \\
72.07 \pm 15.10\end{array}$ & $\begin{array}{c}6.99 \\
(.001) \\
\mathrm{a}>\mathrm{b}, \mathrm{c}\end{array}$ & $\begin{array}{l}72.84 \pm 9.52 \\
71.26 \pm 11.50 \\
71.64 \pm 12.52\end{array}$ & $\begin{array}{l}0.32 \\
(.727)\end{array}$ & $\begin{array}{l}145.50 \pm 21.55 \\
137.19 \pm 25.15 \\
131.07 \pm 22.25\end{array}$ & $\begin{array}{l}2.68 \\
(.071)\end{array}$ \\
\hline $\begin{array}{l}\text { Self-perceived } \\
\text { health }\end{array}$ & $\begin{array}{l}\text { Very healthy } \\
\text { Moderate }^{\mathrm{b}} \\
\text { Poor }^{\mathrm{c}}\end{array}$ & $\begin{array}{r}95 \\
73 \\
4\end{array}$ & $\begin{array}{l}85.27 \pm 15.45 \\
79.41 \pm 14.07 \\
73.50 \pm 26.76\end{array}$ & $\begin{array}{l}3.81 \\
(.024) \\
a>b\end{array}$ & $\begin{array}{l}73.21 \pm 11.26 \\
70.36 \pm 10.55 \\
60.25 \pm 6.95\end{array}$ & $\begin{array}{l}3.67 \\
(.027)\end{array}$ & $\begin{array}{l}144.56 \pm 22.48 \\
132.99 \pm 23.22 \\
109.00 \pm 42.83\end{array}$ & $\begin{array}{c}8.44 \\
(<.001) \\
a>b, c\end{array}$ \\
\hline $\begin{array}{r}\text { Being alone } \\
\text { (min/day) }\end{array}$ & $\begin{array}{l}<119 \\
\geq 120\end{array}$ & $\begin{array}{r}145 \\
27\end{array}$ & $\begin{array}{l}82.57 \pm 15.84 \\
82.22 \pm 13.11\end{array}$ & $\begin{array}{l}0.11 \\
(.905)\end{array}$ & $\begin{array}{l}72.57 \pm 10.86 \\
67.00 \pm 11.22\end{array}$ & $\begin{array}{l}2.44 \\
(.016)\end{array}$ & $\begin{array}{l}138.76 \pm 24.52 \\
139.15 \pm 23.51\end{array}$ & $\begin{array}{l}0.08 \\
(.939)\end{array}$ \\
\hline $\begin{array}{l}\text { Conversing } \\
\text { with parents } \\
\text { (min/day) }\end{array}$ & $\begin{array}{l}<30^{\mathrm{a}} \\
30 \sim 59^{\mathrm{b}} \\
\geq 60^{\mathrm{c}}\end{array}$ & $\begin{array}{l}33 \\
53 \\
86\end{array}$ & $\begin{array}{l}69.70 \pm 16.32 \\
79.25 \pm 12.17 \\
89.44 \pm 12.96\end{array}$ & $\begin{array}{c}28.04 \\
(<.001) \\
\mathrm{a}<\mathrm{b}<\mathrm{c}\end{array}$ & $\begin{array}{l}68.15 \pm 12.22 \\
69.57 \pm 9.55 \\
74.37 \pm 10.94\end{array}$ & $\begin{array}{c}5.46 \\
(.005) \\
a, b<c\end{array}$ & $\begin{array}{l}122.00 \pm 20.83 \\
134.51 \pm 24.22 \\
147.93 \pm 21.46\end{array}$ & $\begin{array}{c}17.67 \\
(<.001) \\
a<b<c\end{array}$ \\
\hline $\begin{array}{c}\text { Internet use } \\
\text { (min/day) }\end{array}$ & $\begin{array}{l}<60^{\mathrm{a}} \\
60 \sim 119^{\mathrm{b}} \\
\geq 120^{\mathrm{c}}\end{array}$ & $\begin{array}{l}79 \\
54 \\
39\end{array}$ & $\begin{array}{l}85.46 \pm 14.97 \\
81.65 \pm 14.46 \\
77.74 \pm 16.56\end{array}$ & $\begin{array}{l}3.49 \\
(.033) \\
a>c\end{array}$ & $\begin{array}{l}74.66 \pm 10.79 \\
69.87 \pm 10.97 \\
68.23 \pm 10.45\end{array}$ & $\begin{array}{c}5.78 \\
(.004) \\
a>b, c\end{array}$ & $\begin{array}{l}139.95 \pm 24.64 \\
138.15 \pm 23.69 \\
137.46 \pm 24.96\end{array}$ & $\begin{array}{l}0.17 \\
(.848)\end{array}$ \\
\hline $\begin{array}{l}\text { Exercises frequency } \\
\text { (per week) }\end{array}$ & $\begin{array}{l}<1 \\
1 \sim 2 \\
3 \sim 4 \\
\geq 5\end{array}$ & $\begin{array}{l}20 \\
38 \\
62 \\
52\end{array}$ & $\begin{array}{l}79.15 \pm 20.42 \\
82.79 \pm 15.37 \\
81.52 \pm 13.72 \\
84.79 \pm 15.27\end{array}$ & $\begin{array}{l}0.78 \\
(.505)\end{array}$ & $\begin{array}{l}72.80 \pm 14.65 \\
72.08 \pm 11.27 \\
69.97 \pm 9.86 \\
73.06 \pm 10.79\end{array}$ & $\begin{array}{l}0.85 \\
(.471)\end{array}$ & $\begin{array}{l}134.25 \pm 24.98 \\
135.08 \pm 23.70 \\
135.76 \pm 25.04 \\
146.96 \pm 22.19\end{array}$ & $\begin{array}{l}2.90 \\
(.036)\end{array}$ \\
\hline
\end{tabular}

${ }^{*} p<.050 ;{ }^{\dagger}$ Included no response. 
ported 30 59 min/day and $<30 \mathrm{~min} /$ day. Family strength also differed significantly by the amount of time spent using the internet $(\mathrm{F}=3.49, p=.033)$. The Scheffé test found that those who reported $<60 \mathrm{~min} /$ day of internet use had significantly higher family strength scores than those who reported $\geq 120$ $\min /$ day.

Several significant differences were found in mean selfcontrol scores according to general characteristics. Sixth-graders scored significantly higher for self-control than fifth-graders $(t=4.10, p<.001)$. Children whose fathers were college graduates or had a higher education level scored significantly higher for self-control than those whose fathers were high school graduates or lower $(t=2.33, p=.021)$. Self-control showed statistically significant difference according to self-perceived health ( $\mathrm{F}=3.67, p=.027)$, time spent alone $(\mathrm{F}=2.44, p=.016)$, and time spent conversing with parents $(\mathrm{F}=5.46, p=.005)$. The Scheffé test found that those who spent $\geq 60 \mathrm{~min} /$ day conversing with parents had higher self-control scores than those who spent $<30 \mathrm{~min} /$ day and 30 59 $\mathrm{min} /$ day conversing with their parents. Self-control significantly differed according to the amount of time spent using the internet $(\mathrm{F}=5.78, p=.004)$. The Scheffé test found that those who reported $<60 \mathrm{~min} /$ day of internet use had higher self-control scores than those who reported 60 119 $\mathrm{min} /$ day or $\geq 120 \mathrm{~min} /$ day.

The following differences were found in the mean happiness score by general characteristics. Sixth-graders scored significantly higher for happiness than fifth-graders $(\mathrm{t}=2.68, p=$ .008). Happiness differed statistically significantly by self-perceived health $(\mathrm{F}=8.44, p<.001)$. The Scheffe test found that children who perceived themselves as very healthy had higher happiness scores than those whose self-perceived health was moderate or poor. Happiness differed significantly by the amount of time spent conversing with parents ( $\mathrm{F}=17.67, p<$ .001). The Scheffe test found that happiness was highest for those spent $\geq 60 \mathrm{~min} /$ day conversing with their parents, followed by those who reported spending 30 59 minutes and $<30 \mathrm{~min} /$ day conversing with their parents. Happiness differed significantly by the frequency of exercise per week ( $\mathrm{F}=$ $2.90, p=.036)$.

The differences in the mean scores of sub-domains of happiness (self-esteem, optimism, peer relations, and home environment) according to general characteristics are presented in Table 2. For self-esteem, sixth-graders scored significantly higher than fifth-graders $(t=3.18, p=.002)$. Self-esteem differed significantly by self-perceived health $(\mathrm{F}=7.90, p=.001)$. The Scheffé test found that children who perceived themselves to be very healthy had higher self-esteem scores than those whose self-perceived health was moderate or poor. Selfesteem differed significantly by the time spent conversing with parents $(\mathrm{F}=12.02, p<.001)$. The Scheffé test found that those who spent $\geq 60 \mathrm{~min} /$ day conversing with their parents had significantly higher self-esteem scores than those who spent $<30 \mathrm{~min} /$ day and 30 59 $\mathrm{min} /$ day conversing with their parents. Self-esteem differed statistically significantly by exercise frequency ( $F=5.26, p=.002)$. The Scheffé test found that participants who exercised $\geq 5$ times a week showed higher self-esteem than those who exercised less than 1, 1 2, and $3 \sim 4$ times a week.

The following differences in mean scores for optimism according to general characteristics were found. Optimism differed statistically significantly by self-perceived health $(\mathrm{F}=9.66, p<.001)$. The Scheffé test found that optimism was highest among those who perceived themselves to be very healthy, followed by those with moderate and poor self-perceived health. Optimism differed significantly by the time spent conversing with parents $(\mathrm{F}=14.21, p<.001)$. The Scheffé test found that optimism was highest among those who spent $\geq 60 \mathrm{~min} /$ day conversing with their parents, followed by those who spent $30 \sim 59 \mathrm{~min} /$ day and $<30 \mathrm{~min} /$ day conversing with their parents.

Multiple significant differences were found in the mean scores for peer relations according to general characteristics. Sixth-graders scored significantly higher than fifth-graders $(\mathrm{t}=3.04, p=.003)$. Peer relations differed statistically significantly by self-perceived health $(\mathrm{F}=5.67, p=.004)$. The Scheffé test found that children who perceived themselves as very healthy scored higher for peer relations than those whose self-perceived health was poor. Scores for peer relations differed significantly by the time spent conversing with parents $(\mathrm{F}=15.61, p<.001)$. The Scheffé test found that scores for peer relations were highest among those who spent $\geq 60 \mathrm{~min} /$ day conversing with their parents, followed by those who spent $30 \sim 59 \mathrm{~min} /$ day and $<30 \mathrm{~min} /$ day conversing with their parents.

The following differences were found in the mean scores for home environment according to general characteristics: Home environment differed statistically significantly by household economic status ( $\mathrm{t}=6.89, p=.001)$ and by self-perceived health $(\mathrm{F}=5.60, p=.004)$. The Scheffé test found that children who perceived themselves as very healthy had higher home environment scores than those with moderate self-perceived health. Home environment differed significantly by the time spent conversing with parents ( $\mathrm{F}=19.90, p<.001)$. The Scheffé test found the highest score for home environment were found among those who spent $\geq 60 \mathrm{~min} /$ day conversing with their parents, followed by those who spent 30 59 $\mathrm{min} /$ day and $<30 \mathrm{~min} /$ day conversing with their parents.

In particular, self-perceived health and time spent conversing with parents were associated with significant differences for all the areas (self-esteem, optimism, peer relations and home environment) (Table 2). 
Table 2. The Sub-domains of Happiness according to General Characteristics

$(N=172)$

\begin{tabular}{|c|c|c|c|c|c|c|c|c|c|c|}
\hline \multirow[b]{2}{*}{ Variables } & \multirow[b]{2}{*}{ Categories } & \multirow[b]{2}{*}{$\mathrm{n}$} & \multicolumn{2}{|c|}{ Self-esteem } & \multicolumn{2}{|c|}{ Optimism } & \multicolumn{2}{|c|}{ Peer relations } & \multicolumn{2}{|c|}{ Home environment } \\
\hline & & & $\mathrm{M} \pm \mathrm{SD}$ & $\begin{array}{l}\text { tor } \mathrm{F}(p) \\
\text { Scheffé }^{*}\end{array}$ & $\mathrm{M} \pm \mathrm{SD}$ & $\begin{array}{l}\text { t or F }(p) \\
\text { Scheffé }^{*}\end{array}$ & $\mathrm{M} \pm \mathrm{SD}$ & $\begin{array}{l}\text { t or F }(p) \\
\text { Scheffé* }\end{array}$ & $\mathrm{M} \pm \mathrm{SD}$ & $\begin{array}{l}\text { t or F }(p) \\
\text { Scheffé* }\end{array}$ \\
\hline Gender & $\begin{array}{l}\text { Male } \\
\text { Female }\end{array}$ & $\begin{array}{r}66 \\
106\end{array}$ & $\begin{array}{l}44.11 \pm 8.11 \\
44.01 \pm 8.41\end{array}$ & $\begin{array}{l}0.07 \\
(.941)\end{array}$ & $\begin{array}{l}40.55 \pm 7.46 \\
40.04 \pm 6.98\end{array}$ & $\begin{array}{l}0.45 \\
(.652)\end{array}$ & $\begin{array}{l}23.27 \pm 4.39 \\
22.95 \pm 4.69\end{array}$ & $\begin{array}{l}0.45 \\
(.656)\end{array}$ & $\begin{array}{l}32.06 \pm 6.24 \\
31.09 \pm 6.14\end{array}$ & $\begin{array}{l}1.00 \\
(.320)\end{array}$ \\
\hline Grade & $\begin{array}{l}\text { Fifth } \\
\text { Sixth }\end{array}$ & $\begin{array}{r}101 \\
71\end{array}$ & $\begin{array}{l}42.41 \pm 8.60 \\
46.38 \pm 7.22\end{array}$ & $\begin{array}{l}3.18 \\
(.002)\end{array}$ & $\begin{array}{l}39.42 \pm 7.21 \\
41.39 \pm 6.95\end{array}$ & $\begin{array}{l}1.80 \\
(.074)\end{array}$ & $\begin{array}{l}22.21 \pm 4.78 \\
24.31 \pm 3.96\end{array}$ & $\begin{array}{l}3.04 \\
(.003)\end{array}$ & $\begin{array}{l}30.70 \pm 6.54 \\
32.55 \pm 5.49\end{array}$ & $\begin{array}{l}1.94 \\
(.054)\end{array}$ \\
\hline $\begin{array}{l}\text { Father's } \\
\text { education }^{\dagger}\end{array}$ & $\begin{array}{l}\text { Hig } \\
\geq \mathrm{C}\end{array}$ & $\begin{array}{l}52 \\
89\end{array}$ & $\begin{array}{l}44.52 \pm 8.43 \\
45.74 \pm 7.80\end{array}$ & $\begin{array}{c}0.87 \\
(.385)\end{array}$ & $\begin{array}{l}40.96 \pm 7.28 \\
40.82 \pm 6.89\end{array}$ & $\begin{array}{l}0.12 \\
(.909)\end{array}$ & $\begin{array}{l}22.87 \pm 5.15 \\
23.88 \pm 4.13\end{array}$ & $\begin{array}{l}1.21 \\
(.230)\end{array}$ & $\begin{array}{l}32.08 \pm 5.76 \\
32.38 \pm 6.09\end{array}$ & $\begin{array}{l}0.29 \\
(.770)\end{array}$ \\
\hline $\begin{array}{l}\text { Mother's } \\
\text { education }^{\dagger}\end{array}$ & $\begin{array}{l}\text { High school } \\
\geq \text { College }\end{array}$ & $\begin{array}{l}58 \\
82\end{array}$ & $\begin{array}{l}44.40 \pm 8.39 \\
45.76 \pm 7.96\end{array}$ & $\begin{array}{l}0.97 \\
(.332)\end{array}$ & $\begin{array}{l}40.28 \pm 6.44 \\
41.16 \pm 7.44\end{array}$ & $\begin{array}{l}0.73 \\
(.466)\end{array}$ & $\begin{array}{l}22.95 \pm 4.85 \\
23.90 \pm 4.30\end{array}$ & $\begin{array}{l}1.23 \\
(.222)\end{array}$ & $\begin{array}{l}31.40 \pm 5.46 \\
32.90 \pm 6.19\end{array}$ & $\begin{array}{l}1.49 \\
(.139)\end{array}$ \\
\hline $\begin{array}{l}\text { Economic } \\
\text { status }\end{array}$ & $\begin{array}{l}\text { High }^{a} \\
\text { Middle }^{b} \\
\text { Low }^{c}\end{array}$ & $\begin{array}{r}44 \\
114 \\
14\end{array}$ & $\begin{array}{l}46.05 \pm 7.06 \\
43.38 \pm 8.81 \\
43.21 \pm 6.51\end{array}$ & $\begin{array}{l}1.74 \\
(.178)\end{array}$ & $\begin{array}{l}41.77 \pm 6.84 \\
39.85 \pm 7.23 \\
38.50 \pm 7.18\end{array}$ & $\begin{array}{l}1.60 \\
(.204)\end{array}$ & $\begin{array}{l}24.02 \pm 4.39 \\
22.81 \pm 4.72 \\
22.29 \pm 3.56\end{array}$ & $\begin{array}{l}1.36 \\
(.260)\end{array}$ & $\begin{array}{l}33.66 \pm 5.57 \\
31.16 \pm 6.11 \\
27.07 \pm 6.13\end{array}$ & $\begin{array}{l}6.89 \\
(.001)\end{array}$ \\
\hline $\begin{array}{l}\text { Self-perceived } \\
\text { health }\end{array}$ & $\begin{array}{l}\text { Very healthy } \\
\text { Moderate }^{\mathrm{b}} \\
\text { Poor }^{\mathrm{c}}\end{array}$ & $\begin{array}{r}95 \\
73 \\
4\end{array}$ & $\begin{array}{c}45.97 \pm 7.57 \\
42.07 \pm 8.19 \\
34.50 \pm 12.77\end{array}$ & $\begin{array}{c}7.90 \\
(.001) \\
a>b, c\end{array}$ & $\begin{array}{l}41.88 \pm 6.37 \\
38.67 \pm 7.08 \\
29.50 \pm 11.96\end{array}$ & $\begin{array}{c}9.66 \\
(<.001) \\
a>b>c\end{array}$ & $\begin{array}{l}23.91 \pm 4.41 \\
22.29 \pm 4.13 \\
17.75 \pm 9.81\end{array}$ & $\begin{array}{l}5.67 \\
(.004) \\
a>c\end{array}$ & $\begin{array}{l}32.80 \pm 5.80 \\
29.96 \pm 6.15 \\
27.25 \pm 8.96\end{array}$ & $\begin{array}{l}5.60 \\
(.004) \\
a>b\end{array}$ \\
\hline $\begin{array}{r}\text { Being alone } \\
\text { (min/day) }\end{array}$ & $\begin{array}{l}<119 \\
\geq 120\end{array}$ & $\begin{array}{r}145 \\
27\end{array}$ & $\begin{array}{l}40.08 \pm 8.39 \\
43.89 \pm 7.77\end{array}$ & $\begin{array}{l}0.11 \\
(.915)\end{array}$ & $\begin{array}{l}40.19 \pm 7.24 \\
40.44 \pm 6.83\end{array}$ & $\begin{array}{l}0.17 \\
(.821)\end{array}$ & $\begin{array}{l}23.04 \pm 4.67 \\
23.26 \pm 4.11\end{array}$ & $\begin{array}{l}0.23 \\
(.821)\end{array}$ & $\begin{array}{l}31.45 \pm 6.21 \\
31.56 \pm 6.14\end{array}$ & $\begin{array}{l}0.08 \\
(.934)\end{array}$ \\
\hline $\begin{array}{l}\text { Conversing } \\
\text { with parents } \\
\text { (min/day) }\end{array}$ & $\begin{array}{l}<30^{\mathrm{a}} \\
30 \sim 59^{\mathrm{b}} \\
\geq 60^{\mathrm{c}}\end{array}$ & $\begin{array}{l}33 \\
53 \\
86\end{array}$ & $\begin{array}{l}39.24 \pm 7.47 \\
42.74 \pm 8.08 \\
46.70 \pm 7.72\end{array}$ & $\begin{array}{l}12.02 \\
(<.001) \\
a, b<c\end{array}$ & $\begin{array}{l}36.09 \pm 6.92 \\
38.66 \pm 7.37 \\
42.79 \pm 6.07\end{array}$ & $\begin{array}{c}14.21 \\
(<.001) \\
\mathrm{a}<\mathrm{b}<\mathrm{c}\end{array}$ & $\begin{array}{l}20.00 \pm 4.68 \\
22.40 \pm 4.81 \\
24.67 \pm 3.61\end{array}$ & $\begin{array}{c}15.61 \\
(<.001) \\
a<b<c\end{array}$ & $\begin{array}{l}26.67 \pm 5.87 \\
30.72 \pm 5.34 \\
33.77 \pm 5.64\end{array}$ & $\begin{array}{c}19.90 \\
(<.001) \\
\mathrm{a}<\mathrm{b}<\mathrm{c}\end{array}$ \\
\hline $\begin{array}{l}\text { Internet use } \\
\text { (min/day) }\end{array}$ & $\begin{array}{l}<60 \\
60 \sim 119 \\
\geq 120\end{array}$ & $\begin{array}{l}79 \\
54 \\
39\end{array}$ & $\begin{array}{l}44.53 \pm 8.24 \\
44.04 \pm 8.10 \\
43.08 \pm 8.69\end{array}$ & $\begin{array}{c}0.40 \\
(.670)\end{array}$ & $\begin{array}{l}40.28 \pm 7.49 \\
40.06 \pm 6.86 \\
40.38 \pm 7.04\end{array}$ & $\begin{array}{l}0.03 \\
(.974)\end{array}$ & $\begin{array}{l}23.37 \pm 4.48 \\
23.06 \pm 4.03 \\
22.51 \pm 5.43\end{array}$ & $\begin{array}{l}0.45 \\
(.636)\end{array}$ & $\begin{array}{l}31.77 \pm 6.28 \\
31.00 \pm 6.43 \\
31.49 \pm 5.73\end{array}$ & $\begin{array}{l}0.25 \\
(.780)\end{array}$ \\
\hline $\begin{array}{l}\text { Exercises } \\
\text { frequency } \\
\text { (per week) }\end{array}$ & $\begin{array}{l}<1^{\mathrm{a}} \\
1 \sim 2^{\mathrm{b}} \\
3 \sim 4^{\mathrm{c}} \\
\geq 5^{\mathrm{d}}\end{array}$ & $\begin{array}{l}20 \\
38 \\
62 \\
52\end{array}$ & $\begin{array}{l}41.15 \pm 9.03 \\
42.08 \pm 7.69 \\
43.23 \pm 8.25 \\
47.58 \pm 7.42\end{array}$ & $\begin{array}{c}5.26 \\
(.002) \\
a, b, c<d\end{array}$ & $\begin{array}{l}39.60 \pm 6.85 \\
39.76 \pm 7.37 \\
39.16 \pm 7.66 \\
42.10 \pm 6.27\end{array}$ & $\begin{array}{l}1.77 \\
(.155)\end{array}$ & $\begin{array}{l}22.90 \pm 4.95 \\
22.32 \pm 4.20 \\
22.55 \pm 4.60 \\
24.33 \pm 4.51\end{array}$ & $\begin{array}{l}1.97 \\
(.121)\end{array}$ & $\begin{array}{l}30.60 \pm 6.44 \\
30.92 \pm 6.34 \\
30.82 \pm 6.34 \\
32.96 \pm 5.67\end{array}$ & $\begin{array}{l}1.48 \\
(.222)\end{array}$ \\
\hline
\end{tabular}

${ }^{*} p<.050 ;{ }^{\dagger}$ Included no response.

\section{Correlations among Family Strength, Self-control, and Happiness of Participants}

The correlations among family strength, self-control, and happiness are presented in Table 3. Happiness was significantly positively correlated with family strength $(\mathrm{r}=.78, p<$ $.001)$ and self-control $(\mathrm{r}=.59, p<.001)$, and family strength was significantly positively correlated with self-control $(\mathrm{r}=.55, p<$ .001).

Family strength was significantly positively correlated with the pursuit of long-term $(\mathrm{r}=.59, p<.001)$ and immediate satisfaction $(r=.32, p<.001)$ among the sub-domains of self-control. Family strength was significantly positively correlated with self-esteem $(\mathrm{r}=.73, p<.001)$, optimism $(\mathrm{r}=.70, p<.001)$, peer relations $(\mathrm{r}=.69, p<.001)$, and a home environment $(\mathrm{r}=.79, p<$ .001) among the sub-domains of happiness.

Self-control was significantly positively correlated with family resilience $(\mathrm{r}=.53, p<.001)$, mutual respect and acceptance $(\mathrm{r}=.54, p<.001)$, qualitative bonding $(\mathrm{r}=.49, p<.001)$, economic stability and cooperation $(\mathrm{r}=.36, p<.001)$, and family cultural and social participation $(\mathrm{r}=.39, p<.001)$ among the sub-domains of family strength. Self-control was significantly positively correlated with self-esteem $(\mathrm{r}=.62, p<.001)$, optimism $(\mathrm{r}=.50, p<.001)$, peer relations $(\mathrm{r}=.53, p<.001)$, and home environment $(\mathrm{r}=.53, p<.001)$ among the sub-domains of happiness.

Happiness was significantly positively correlated with family resilience $(\mathrm{r}=.77, p<.001)$, mutual respect and acceptance $(\mathrm{r}=.79, p<.001)$, qualitative bonding $(\mathrm{r}=.68, p<.001)$, economic stability and cooperation $(\mathrm{r}=.53, p<.001)$, and family cultural and social participation $(\mathrm{r}=.56, p<.001)$ among the sub-domains of family strength. Happiness was also significantly positively correlated with the pursuit of long-term $(\mathrm{r}=.67, p<.001)$ and immediate satisfaction $(\mathrm{r}=.31, p<.001)$ among the sub-do- 
mains of self-control. Higher levels of family strength and selfcontrol were correlated with higher levels of happiness.

\section{Factors Affecting Happiness of Participants}

The variable inflation factor (VIF) and tolerance were estimated to determine multicollinearity among the independent variables used in the regression analysis. There was no multicollinearity, with - VIF values of 1.02 1.46 and tolerance values of .69 .98. There was no auto-correlation, as shown by a Durbin-Watson statistic of 1.72. Therefore, the assumptions for the regression analysis were met. The regression analysis of happiness was conducted using family strength, self-control, weekly exercise frequency, and self-perceived health as independent variables (Table 4). The stepwise regression analysis model for identifying the factors affecting the children's happiness was statistically significant $(\mathrm{F}=74.23, p<.001)$.
The factors affecting happiness among late school-aged children were family strength $(\beta=.63, p<.001)$, self-control $(\beta=$ $.21, p<.001)$, exercising $\geq 5$ times a week $(\beta=.15, p<.001)$, and poor or moderate self-perceived health $(\beta=-.12, p=.007 ; \beta=$ $-.09, p=.040$, respectively). These factors accounted for $69 \%$ of the total variance. The children's happiness was primarily explained by family strength $(61 \%)$ as well as by self-control (4\%). A smaller effect was observed for exercising $\geq 5$ times a week (2\%). Late school-aged children with higher levels of family strength and self-control felt happier. Happiness was higher in those exercising $\geq 5$ times a week, and was lower in those with poor or moderate self-perceived health.

\section{DISCUSSION}

A healthy family is characterized by mutual respect and acceptance, qualitative bonding, economic stability and cooper-

Table 3. Correlations among Family Strength, Self-control, and Happiness

$(N=172)$

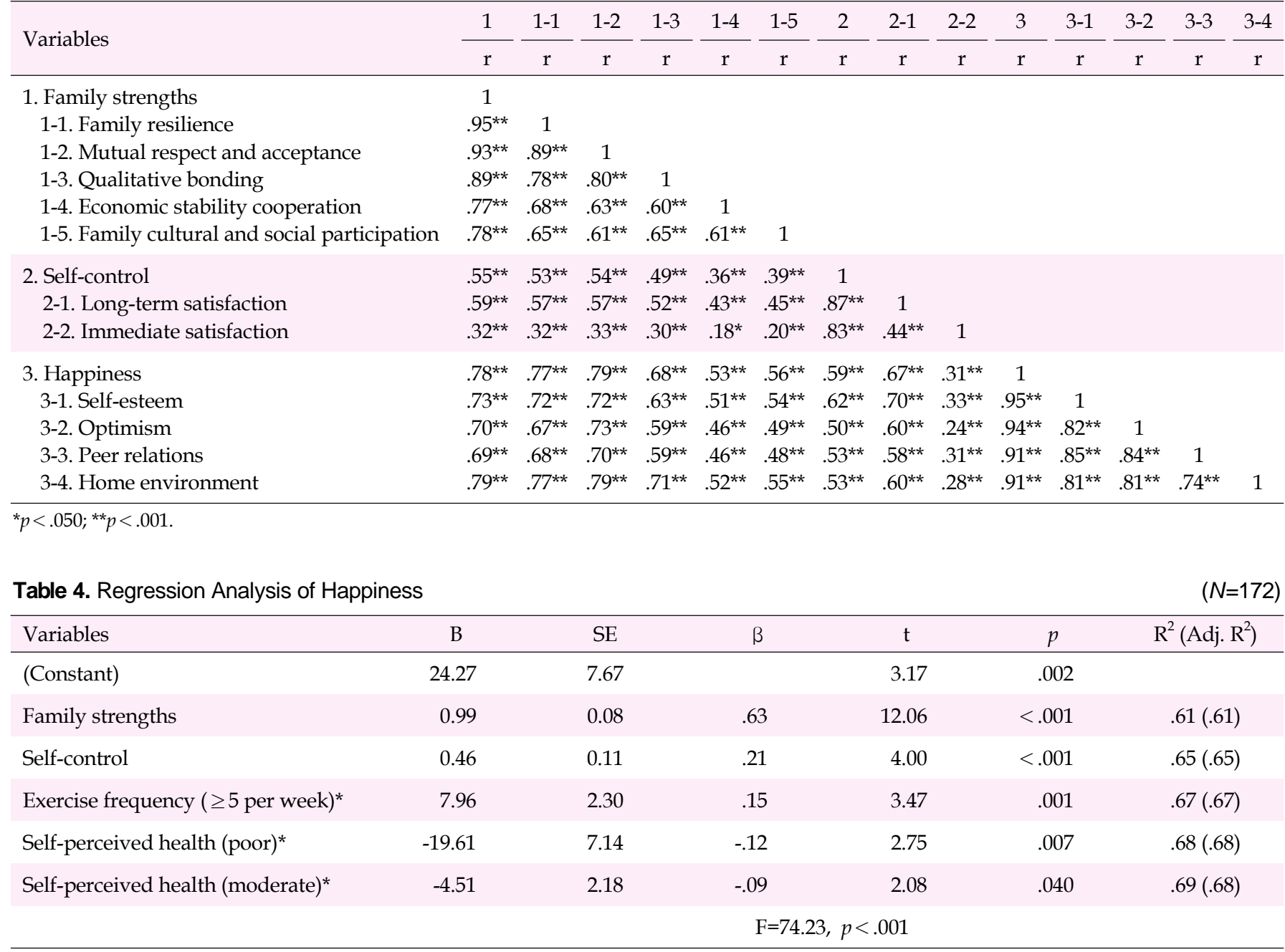

*Dummy variables. 
ation, family elasticity, and family cultural and social participation [13]. This study found that family strength was associated with high economic status and self-perceived health. Family strength was also higher in respondents who reported spending a small amount of time using the internet and a large amount conversing with their parents. Economic stability is an important element of basic family life. It is therefore believed that higher economic status leads to greater family strength. Those regarding themselves as healthy had higher levels of family strength. The physical health of family members is one of the characteristics of a healthy family [10]. It is therefore necessary to expand education on positive health perceptions and physical activity programs in order to improve family strength. In this study, high family strength was inversely correlated with internet usage time and directly correlated with time spent conversing with parents. School-aged students in Korea were found to use the internet for more than 30 minutes and less than 1 hour per day (41.7\%) [24]. The result in the present study indicates that time spent in convertsation-as compared to personal online activity-promotes family strength. Therefore, it is necessary to ensure time for convertsation with parents as a means of improving family strength, and such an approach is expected to improve family strength through experiential learning programs for late school-aged children.

The children's score for self-control was higher than in a previous study using the same tool [18]. Research on self-control related to problem behaviors has been conducted at national and international levels [22,26-28], and improvements in self-control have been found due to an increasing recognition of its importance. In this study, those who spent less time alone and on the internet, and more time conversing with their parents, had higher self-control. This reflects the process of self-control formation, in which self-control is internalized through repetition and reinforcement of external control [17]. That is, late school-aged children who have more chances to receive and internalize advice from influential people around them develop better self-control.

This observation is also consistent with the finding that problematic internet utilization was correlated with low self-control [24,27]. A literature review regarding internet addiction found that lower scores for self-control were correlated with internet addiction and longer internet usage time [24]. While it is necessary to conduct further research on this topic because studies have used different scales for internet usage time, improved self-control is expected to reduce internet addiction and internet usage time. Children with better self-control used the internet and smartphones with greater moderation, and it is likely that they have less exposure to problem behaviors and problematic internet and smartphone utilization than those with poor self-control. High self-control was associated with spending more time conversing with parents. This result is similar to the finding that adolescents who engaged in less conversation with their parents were more likely to adhere to peer relations and had a higher likelihood of experiencing problem behaviors [27]. Time spent conversing with parents also makes a difference in children's self-control.

In this study, sixth-graders were happier than fifth graders, and high self-perceived health and more time spent conversing with parents were also associated with happiness among school-age children. Regarding the finding of this study that sixth-graders felt happier, regardless of gender, previous research comparing elementary and middle school students [29] found that higher-grade elementary school students felt happier. Happiness by school grade differed among the children; therefore, further research should be conducted on happiness by grade in school. The children who perceived themselves as very healthy were happier than their counterparts. This result is consistent with the previous finding that elementary school children with better subjective health status felt happier, were more satisfied, and had fewer negative emotions [23]. As mentioned above, physically healthy children seemed to feel happier. Happiness has the same meaning as subjective well-being and satisfaction, and those regarding themselves as healthy are expected to feel more satisfied and happier. Happiness was higher among children who spent more time conversing with their parents. This result is consistent with the finding that better intra-family conversations and higher levels of family support, were associated with happiness [29]. Time spent conversing with parents made significant differences in late school-aged children's happiness, as well as in family strength and self-control. Therefore, if late school-aged children can ensure that they spend more than 60 minutes per day conversing with their parents, they can increase their happiness by improving family strength and self-control.

Late school-aged children's happiness did not differ by economic status, in contrast to the previous finding that social class and family income were factors affecting happiness [8]. Economic status made significant differences in family strength and home environment among the sub-areas of happiness, but did not affect self-esteem, optimism, or peer relations. It is therefore difficult to say that household economic status impacts happiness. Late school-aged children with greater family strength were happier. This is consistent with previous studies of family strength in preschoolers [29] and schoolaged children [30]. It is believed that school-aged children can perceive support and satisfaction as members of society and feel happy when they experience healthy and good human 
relationships through a healthy family [1]. Family strength accounted for $61 \%$ of children's happiness. Self-control accounted for additional $4 \%$ of children's happiness. Children with better self-control felt happier. This result shows that school-aged children's happiness was correlated with their pursuit of long-term satisfaction. Pursuit of long-term satisfaction is expected to be an important engine that enables school-aged children experiencing long-term school life to be mentally healthy. Furthermore, exercising $\geq 5$ times per week exerted a smaller, but still meaningful, effect on happiness, with an explanatory power of $2 \%$.

Numerous studies have investigated ways to reduce depressive factors and to prevent addiction or problem behaviors, with the objective of improving happiness among children. Based on this study, more attempts should be made to make children happier by improving family strength, by reinforcing self-control and positive health perceptions, and by improving physical activity. This study is distinct from those that have sought to explain children's happiness in terms of school-related variables, such as performance and adjustment to school. To increase the happiness of school-aged children, it is necessary to expand the scope of nursing into community-based health education, including physical activity programs at school and at home and interventions designed to foster positive health perceptions, instead of limiting interventions to personal factors. This study is significant in that it provided evidence for the importance of family strength for school-aged children and furnished basic data suggesting interventions to make them happier by fostering positive mental habits.

\section{CONCLUSION}

In today's society, the intense demands of academic work and high levels of stress are threatening children's mental health. Suicide is the top cause of death among adolescents in Korea. This study aimed to investigate family strength, selfcontrol, and happiness, as perceived by school-aged children, and on this basis, to obtain basic data for use in developing a programs to make them happier. Family strength and selfcontrol jointly accounted for $65 \%$ of school-aged children's happiness. Children exercising $\geq 5$ times per week felt happier, and exercise had an explanatory power of $2 \%$ for children's happiness. Therefore, further studies should investigate the role of exercise in greater depth. Furthermore, children who regarded their health as moderate or poor were less likely to feel happy. Therefore, efforts should be made to reinforce family strength and self-control, to encourage more frequent exercise on a weekly basis, and to foster positive health perceptions in order to improve happiness among school-aged children.

The results of this study were specific to the location where it was conducted, making it difficult to generalize our results. Therefore, further research should be conducted with an expanded selection of participants. On the basis of these results, it is necessary to develop and apply programs that reinforce family strength and self-control for school-aged children. Further research should be conducted into other factors influencing late school-aged children's happiness.

\section{Conflict of interest}

No existing or potential conflict of interest relevant to this article was reported.

\section{REFERENCES}

1. Hockenberry MJ, Wilson D, Rodgers CC. Wong's nursing care of infants and children. 11th ed. St Louis, MO: Elsvier Inc.; 2018. p. 8-23.

2. Statistics Korea, Ministry of Gender Equality and Family. Youth statistics in 2016 [Internet]. Daejeon: Statistics Korea; 2015 [cited $2019 \mathrm{Feb} 1]$. Available from:

http://kostat.go.kr/portal/korea/kor_nw/1/6/1/index.board? bmode $=$ read $\& b S e q=\& a S e q=353501 \&$ pageNo $=4 \&$ row $\mathrm{Num}=10 \&$ navCount $=10 \&$ currPg $=\&$ sTarget $=$ title $\& \mathrm{sT} x \mathrm{t}=0$.

3. Vaillant GE. Aging well: Surprising guideposts to a happier life from the landmark Harvard study of adult developmen. 1st ed. Boston: Little, Brown and Company; 2008. p. 9-29.

4. Lawrence EM, Rogers RG, Wadsworth T. Happiness and longevity in the United States. Social Science and Medicine. 2015;145:115-119. https://doi.org/10.1016/j.socscimed.2015.09.020

5. Yeom YS, Kim KM, Lee SW. 2016 8th International comparative study of child and adolescent happiness index. Research Results Report. Seoul: Korea Bang Jung Hwan Foundation; 2016 May.

6. Kang YH. Exploring the constructs of happiness of elementary students. The Journal of Elementary Education. 2008;21(2):159-177.

7. Baumgardner SR, Crothers MK. Positive psychology. 1st ed. United States of Amarica: Pearson; 2009. p. 26-59.

8. Park Y, Kim U. Factors influencing happiness among Korean adolescents: With specific focus on the influence of psychological, relational and financial resources and academic achievement. Korean Journal of Psychological and Social Issues. 2009;15(3):399-429.

9. Otto HA. What is a strong family? Marriage and Family Living. 1962;24(1):77-80. https://doi.org/10.2307/348232

10. Yoo YJ. A discussion on the concept of sound home and healthy family. Journal of Korean Home Management Association. 1999; 17(3):93-102.

11. Yoo GS. A survey on the elements of strong families: Focusing on family systems and family strengths. Journal of Family Relations. 
2004;9(2):25-42.

12. Yoo YJ. A study on the development of Korean family strengths scale for strengthening the family. Journal of Family Relations. 2004;9(2):119-151.

13. Yoo Y, Lee I, Kim S, Choi H. Development of Korea family strengths scale (KFSS-II). Journal of Korean Home Management Association. 2013;31(4):113-129.

https://doi.org/10.7466/JKHMA.2013.31.4.113

14. Ho K, Shaul RZ, Chapman LA, Ford-Jones EL. Standard of care in pediatrics: Integrating family-centred care and social determinants of health. Healthcare Quarterly. 2016;19(1):55-60.

https://doi.org/10.12927/hcq.2016.24608

15. Lee GN. A review study of self-control in children. Dong-A Educational Review. 1995;21:103-124.

16. Kim WH, Lee DH. The relationships among perceived interparental conflict and self-control, school mal-adjustment behaviors in middle school students. Korean Journal of Counseling. 2010;11(4): 1767-1782.

17. Burns CE, Dunn AM, Brady MA, Starr NB, Blosser CG. Pediatric primary care. 4th ed. St Louis, MO: Elsvier Inc.; 2009. p. 73-91.

18. Nam HM, Ok SW. The effects of psychological family environment, self-control and friend characteristics of middle school students on their problem behaviors. Family and Environment Research. 2001;39(7):37-58.

19. Cottrell L. Joy and happiness: A simultaneous and evolutionary concept analysis. Journal of Advanced Nursing. 2016;72(7):1506-1517. https://doi.org/10.1111/jan.12980

20. Hockenberry M, Wilson D. Wong's essentials of pediatric nursing. 9th ed. St Louis, MO: Elsvier Inc.; 2012.

21. Kang H. Effect size and sample size calculation using G*POWER. Paju: Freedom Academy; 2019. p. 105-115.

22. Koo HY. Effects of a cell phone use education program on knowledge, attitude, self-efficacy, and self-control of cell phone use in elementary school students. Journal of Korean Academy of Child Health Nursing. 2012;18(3):109-118.

https://doi.org/10.4094/jkachn.2012.18.3.109

23. Jung Y. Analysis on the variables affecting perceived happiness of elementary school students [dissertation]. Seoul: Ewha Womans University; 2015. p. 1-237.

24. Moon YI, Koo HY, Park HR. Scope of internet addiction and predictors of addiction in Korean children in early elementary school. Korean Journal of Child Health Nursing. 2005;11(3):263-272.

25. Kim IS. The influence of amount of physical activity and exercise participation factors of elementary school children on their happiness. The Korean Journal of Elementary Physical Education. 2011; 17(3):113-123.

26. Park YS, Park JH. The effects of school violence victimization on cyberbullying perpetration in middle school students and the moderating role of self-control. Korean Journal of Child Studies. 2016;37(3):39-51. https:// doi.org/10.5723/kjcs.2016.37.3.39

27. Li D, Li X, Wang Y, Zhao L, Bao Z, Wen F. School connectedness and problematic internet use in adolescents: A moderated mediation model of deviant peer affiliation and self-control. Journal of Abnormal Child Psychology. 2013;41(8):1231-1242. https://doi.org/10.1007/s10802-013-9761-9

28. Yeun YR, Han SJ. Effects of psychosocial interventions for schoolaged children's internet addiction, self-control and self-esteem: Meta-analysis. Healthcare Informatics Research. 2016;22(3):217-230. https://doi.org/10.4258/hir.2016.22.3.217

29. Jo EJ. Impact factors of perceived happiness among primary schoolchild and middle school student from low-income families: Focused on individual factors, family factors, and school factors. Youth Facilities and Environment. 2014;12(1):53-64.

30. Kwon YH. The effects of temperament and family strengths on child's happiness. The Journal of Future Early Childhood Education. 2015;22(2):309-331. 